Journal of Animal and Veterinary Advances 10 (15): 1947-1955, 2011

ISSN: $1680-5593$

(C) Medwell Journals, 2011

\title{
Gene Expression of Metabolizing Enzymes in Beagle Dog Intestine
}

\author{
${ }^{1}$ Sung-Won Park, ${ }^{1} \mathrm{Hee} \mathrm{Yi,}{ }^{1}$ Soo-Min Cho, ${ }^{1}$ Kyul Jo, ${ }^{1} \mathrm{Jin}-\mathrm{A}$ Park, ${ }^{2}$ Soo-Jean Shin, \\ ${ }^{1} \mathrm{Hee}-\mathrm{Jung} \mathrm{Cho},{ }^{3} \mathrm{Si}$-Whan Song, ${ }^{4} \mathrm{Sang}$ Min Jeong and ${ }^{1} \mathrm{Ho}-\mathrm{Chul}$ Shin \\ ${ }^{1}$ Department of Veterinary Pharmacology and Toxicology, Konkuk University, \\ 143-701 Seoul, Republic of Korea \\ ${ }^{2}$ Athens Academy, Athens, GA30606, USA \\ ${ }^{3}$ Preclinical Research Center, Chemon Inc., Yongin, 449-826 Kyunggi-do, Republic of Korea \\ ${ }^{4}$ Department of Biochemistry and Molecular Cell Biology, College of Veterinary Medicine, \\ Konkuk University, 143-701 Seoul, Republic of Korea
}

\begin{abstract}
This study was performed to set up a transcriptional database of the intestinal metabolizing enzymes in beagle dogs. The total RNA was isolated from the duodenum and the mRNA expression was measured using GeneChip ${ }^{\circledast}$ oligonucleotide arrays. Detected genes from the intestine were about $47 \%$ of 43,035 sequences and total of 79 genes involved metabolizing enzymes. Among the phase I enzymes, dogs exhibited abundant gene expressions of CYP3A12, CYP2B11, LOC610195 (similar to CYP2J2) followed by LOC489851 (similar to CYP3A4), CYP27A1 and CYP51. For phase II enzymes, acetyltransferase ACAT1, glutathione S-transferases GSTA3 and GSTP1, sulfotransferases SULT1A1 and SULT1D, acyltransferases DGAT1 and ACAA1 and glucuronosyltransferase UGCG were highly expressed in duodenum. The dogs expression profiles were compared with those in mice based on gene classification and annotation. Between the two species, the regression of all enzymes $(n=36)$ with same annotations was 0.496 as andcoefficient of determination $\left(R^{2}\right)$ however, two cytochrome P450s including CYP2S1 and CYP4B1 were expressed $<5$ fold and phase II enzymes including GSTA3, SULT1A1, SULT1D1, TPST1 and UGCG were expressed $>5$ fold changes in dogs (t-test, $\mathrm{p}<0.01)$. In sum, theses data indicated significant differences between beagle dogs and ICR mice in the mRNA expression of both $\mathrm{p} 450 \mathrm{~s}$ and phase II metabolizing enzymes. These animals are the most widely used species/lines in toxicological and pharmacological screening. Therefore, this database will be useful for predicting and scaling the intestinal drug metabolism between rodents (mice) and non-rodents (dogs).
\end{abstract}

Key words: Gene expression, metabolizing enzymes, intestine, mouse, dog

\section{INTRODUCTION}

Exploration of the genome using DNA microarrays and other genome-scale technologies narrows the gap in the knowledge of gene function and molecular biology between the currently-favored model organisms and other species (Brown and Botstein, 1999). The completion of the genomic sequences of model organisms and the DNA sequencing of human and knowledge of cross-species gene homologies enables to study the different gene expression in animal models (Che et al., 1995). Among the model animals, dogs are recognized as an unrivalled model for the study of human disease (Starkey et al., 2005). Additionally, dogs have been emerged as a premier species for the study of biology and the recent availability of a high-quality draft sequence lifts the dogs system to a new threshold. The dogs genome organization was extensively studied in the last 10 years. With advances in genomics, microarray technology has been used in targeting specific tissue genes such as intestinal transporters and/or enzymes. This type of study has the potential to greatly enhance the understanding of pharmacokinetic and pharmacodynamic properties of oral drug molecules. It is now accepted that the drug absorption process from intestine is highly associated with the functional gene expression of intestinal transporters and metabolizing enzymes (Wang et al., 1999).

After absorption, many drugs are chemically altered in the body by various reactions of metabolizing enzymes which are classically divided into phase I and Phase II enzymes (Anzenbacher and Anzenbacherova, 2001). Although, the enzymes are predominantly found in the liver, the intestinal enzymes also deserve special mechanism for modulating the drug availability as the main contributor of prehepatic clearance of drugs (Beaumont, 2003). Furthermore, the commonly prescribed drugs undergo extensive first-pass metabolism following

Corresponding Author: Ho-Chul Shin, Department of Veterinary Pharmacology and Toxicology, College of Veterinary Medicine, Konkuk University, 143-701 Seoul, Korea 
oral ingestion. For all of these at least $45 \%$ of the original doses were lost on average by the time they reach the systemic circulation. Knowledge of the degree and variation in the expression of the major drug metabolizing enzymes of the intestine is therefore, essential as not only such enzymes represent a key determinant of the extent of first-pass elimination but also the interspecies variation in the oral bioavailability, the probability and the magnitude of drug-drug or food-drug interactions. Recognition of these potential consequences of intestinal metabolism should further aid and predict the oral bioavailability and drug disposition during both in clinical and preclinical stages of development.

The genomic comparison of the intestinal enzymes among some species is very valuable for interspecies comparison of drug bioavailability and animal scaling for predicting the metabolic capacity. Although, mouse and dogs are not used as model animals in classical pharmacology and toxicology screening but also are important species (dogs) in the field of veterinary medicine. Comparative estimation of these species is very limited to the gene expression levels of drug targeting (Amidon et al., 1988). Thus, it is interesting to investigate the transcriptional profiles of the intestinal metabolizing enzymes in animal models for assessing the factors which altering pharmacokinetics (Oh et al., 1993). This study was therefore, carried out to set up a dog gene expression database in duodenum and investigated the effective statistical and bioinformatical method to compare the different data set from different species, tissue types, physiological state and types of DNA microarrays.

\section{MATERIALS AND METHODS}

Materials: TRIzol reagent and superscript choice system for cDNA synthesis kit were purchased from Invitrogen (Carlsbad, USA). BioArray high-yield RNA transcript labeling kit was obtained from Enzo Biochem (New York, USA). RNeasy kit was supplied by Qiagen (Valencia, USA). GeneChips including Dogs 2.0 and mouse 430A 2.0 were provided by Affymetrix (Santa Clara, CA, USA). The GeneChip hybridization and scanning were performed at the Seoulin Molecular Biology Technique Center (Seoul, Republic of Korea).

Animals: Beagle dogs (4 males, 5.5-7.6 kg, Marshall Beijing, China) were housed in a controlled semi-barrier system room of Chemon Co. (Yongin, Republic of Korea). The animals were determined to be healthy on the basis of clinical examination (KFDA' Guide for the Care and Use of Laboratory Animals in 2009). ICR mice (30-40 g) were obtained from Orient Bio Co. (Seoul, Republic of Korea) and housed in a controlled animal room of Konkuk University, Seoul, Republic of Korea. The animals were fed solid pellets and watered ad libitum. All procedures were carried out with the approval of the Konkuk University Institutional Animal Care and Use Committee.

RNA isolation: Mucosal tissues of dog and mouse duodenum were immediately scrapped with a clean glass slide, transferred to a new frozen vial and dipped into liquid $\mathrm{N}_{2}$. Approximately $100 \mathrm{mg}$ of tissue was added to $1 \mathrm{~mL}$ TRIzol reagent and homogenized with razor on ice. The homogenate was transferred to a new tube and then $200 \mu \mathrm{L}$ chloroform was added to the TRIzol mixture. After centrifuged at $12,500 \mathrm{rpm}$ for $15 \mathrm{~min}$ at $4^{\circ} \mathrm{C}$, the aqueous phase was transferred to a new tube and the RNA was precipitated with a $500 \mu \mathrm{L}$ of isopropanol and washed with $80 \%$ ethanol. The RNA was further purified with an RNease Mini kit (Qiagen, Valencia, USA) according to manufacturer's manual. The concentration of the purified RNA was measured at $260 \mathrm{~nm}$. About $5 \mu \mathrm{g}$ of the purified RNA was mixed with RNA loading buffer and heated at $75^{\circ} \mathrm{C}$ for $15 \mathrm{~min}$. After cooling down on ice for $5 \mathrm{~min}$, the RNA was loaded on 1\% agarose/formaldehyde gel in $1 \times$ MOPS buffer. The gel was run at $80-100 \mathrm{~V}$ for $50 \mathrm{~min}$ and two sharp 18 and $28 \mathrm{~S}$ bands was confirmed under UV.

Microarray assay: From the total RNA samples, probe synthesis, hybridization, detection and scanning were performed according to the standard protocols from Affymetrix, Inc. (Santa Clara, USA). The cDNA was synthezied using the One-Cycle cDNA Synthesis Kit (Affymetrix, Santa Clara, CA, USA). Single stranded cDNA was synthesized using Superscript II reverse transcriptase and $\mathrm{T} 7$-oligo $(\mathrm{dT})$ primers at $42^{\circ} \mathrm{C}$ for $1 \mathrm{~h}$. Double stranded cDNA was obtained by using DNA ligase, DNA polymerase I and RNase $\mathrm{H}$ at $16^{\circ} \mathrm{C}$ for $2 \mathrm{~h}$ followed by T4 DNA polymerase at $16^{\circ} \mathrm{C}$ for $5 \mathrm{~min}$. After clean up with a Sample Cleanup Module (Affymetrix, Santa Clara, CA, USA), ds-cDNA was used for in vitro Transcription (IVT). The cDNA was transcribed using the GeneChip IVT Labeling Kit (Affymetrix, Santa Clara, CA, USA) in the presence of biotin-labeled CTP and UTP. After clean up with a Sample Cleanup Module (Affymetrix, Santa Clara, CA, USA), the biotin-labeled IVT-RNA was fragmented. Fragmented cRNA was hybridized at $45^{\circ} \mathrm{C}$ for $16 \mathrm{~h}$ according to the manufacturer's instructions. After hybridization, the arrays were washed in a GeneChip Fluidics Station 450 with a non-stringent wash buffer at $25^{\circ} \mathrm{C}$ followed by a stringent wash buffer at $50^{\circ} \mathrm{C}$. After washing, the arrays were stained with a streptavidin-phycoerythrin complex. After staining, the intensities were determined with a GeneChip scanner. The 
duodenal mRNA expression profile obtained for SLC15A1 from microarray data analyses was validated using semiquantitative RT-PCR. The RT-PCR assay was performed as described previously (Shin et al., 2009). SLC15A1 mRNA expression in the individual biopsies determined by RT-PCR exhibited a pattern similar to that observed with the microarray data.

Data analysis: Official symbols and gene names were used in accordance with the symbol and name lists approved by HUGO (Human Genome Organization) Gene Nomenclature Committee (http://www.genenames.org). Data analysis was performed using GeneSpring 7.2 (Silicon Genetics, Redwood city, CA, USA). Numeric data were extracted from DAT images and normalized using Microarray Suite. Gene function analysis was performed using the gene ontology-mining tool of NetAffx which is based on the Gene Ontology database (http://www. geneontology.org). GeneSpring also uses data found publicly in genomics databases to build gene ontologies based on annotation information. For the present GeneChip probe array study, the data for each genes represents data from 11-20 probe pairs each approximately $25 \mathrm{bp}$ in length. The overall target-specific intensity was obtained by the difference between the intensity of perfect match and the mismatch probes. For normalization, data from each expression array were scaled so that the overall fluorescence intensity across each chip was equivalent (average target intensity set at 500). The One-Side Wilcoxon's Signed Rank test was employed to generate the detection p-value. If the overall intensity of perfect match were much larger than that of mismatch, the detection p-value would be small. The probe set would be regarded as present if the p-value was $<0.04$ and if the p value was $>0.06$, the probe set would be regarded as absent. The change algorithm generated a change p-value and an associated fold-change value. The second algorithm gave a quantitative estimate of the change in gene expression in the form of Signal Log Ratio. The level of gene expression was regarded as increased if its change p-value was $<0.0025$ and the gene expression would be considered to be decreased if its change p-value was $>0.9975$.

\section{RESULTS}

Gene analysis: Totally 43, 035 sequences of genes from dogs duodenum were measured and analyzed and 20, 153 (46.83\%) of them were expressed over 1.0 fold change. The number of expressed enzyme genes was 23 for Cytochrome P450s, 14 for acetyltransferases, 10 for acyltransferases, 2 for glucuronosyltransferases, 18 for
glutathione-S-transferases and 12 for sulfotransferases. Based on gene classification and annotation, the expression levels of drug-metabolizing enzymes in dogs $(\mathrm{n}=4)$ were compared to those in mice $(\mathrm{n}=5)$. In both species, the regression of all enzymes $(n=36)$ with same annotations was 0.496 as coefficient of determination $\left(\mathrm{R}^{2}\right)$. However, two cytochrome P450s including CYP 2S1 and CYP4b1 were expressed in $<5$ fold and phase II enzymes including GSTA3, SULT1A1, SULT1D1, TPST1 andUGCG were expressed in $>5$ fold (t-test, $\mathrm{p}<0.01$ ).

Cytochrome P450s: Figure la-d shows a substantial interspecies variation in the expression levels of Cytochrome P450s, CYP2B11, 2J2, LOC489851, 3A12, 4B1 and $27 \mathrm{~A} 1$ of dogs and CYP2B20, 2C40, 2C55, 2C65, 2D26, $2 \mathrm{~J} 6,3 \mathrm{~A} 11,3 \mathrm{~A} 13,3 \mathrm{~A} 25,4 \mathrm{~F} 14,4 \mathrm{~V} 3$ and $27 \mathrm{Al}$ of mouse were dominantly expressed (expression levels $>10.0$ ). Only CYP2S1, CYP4B1, CYP21A1, CYP27B1 and CYP51 were expressed in both species and were significantly different (expression levels $>1.0$ and t-test, $\mathrm{p}<0.01$ ). Expression levels were measured by the intensity of hybridization signal using GeneChip array; a) CYP1A1 CYP2C70 b) CYP2D10 CYP2W1, c) CYP3A4 CYP4X1, d) CYP7A1 CYP51) (LOC475225: similar to CYP 51A1, LOC476453: similar to CYP2T1, LOC478337: similar to Peptidyl-prolyl cis-trans isomerase $\mathrm{B}$ precursor, LOC478365: similar to CYP11A1, LOC484491: similar to CYP 2 S 1, LOC489816: similar to NADPH-cytochrome P450 reductase, LOC489851: similar to CYP3A4, LOC607548: similar to CYP 4V2, LOC608452: similar CYP 4B1, LOC610195: similar to CYP 2J2, LOC610489: similar to CYP 27, mitochondrial precursor, LOC612477: similar to CYP 2F1).

Acetyltransferases: Acetylation of drug compounds is catalyzed in human and animals by various types of acetyltransferases such as histone acetyltransferases (MYSTs), N-Acetyltransferases (NATs) and spermidine/ spermine N1-acetyltransferases (SATs). In the present study (Fig. 2), ACAT1, ACAT2, ARD1, CRAT, GNPNAT1, MYST4 and NAT5 were commonly expressed and among them ACAT1 was dominant in both species however, no tendency of expression difference was observed. Expression levels were measured by the intensity of hybridization signal using GeneChip array. (ACAT1, 2: Acety-Coenzyme An acetyltransferase 1, 2, ARD1: N-acetyltransferase ARD1 homolog, CRAT: carnitine acetyltransferase, DLAT: Dihydrolipoamide S-acetyltransferase, GCAT: Glycine C-acetyltransferase, GNPNAT1: Glucosamine-phosphate N-acetyltransferase 1, MYST2, 3, 4 : MYST histone acetyltransferase 2,3,4, 4, NAT2, 5, 6: N-acetyltransferase 2, 5, 6, Cast1: O- 

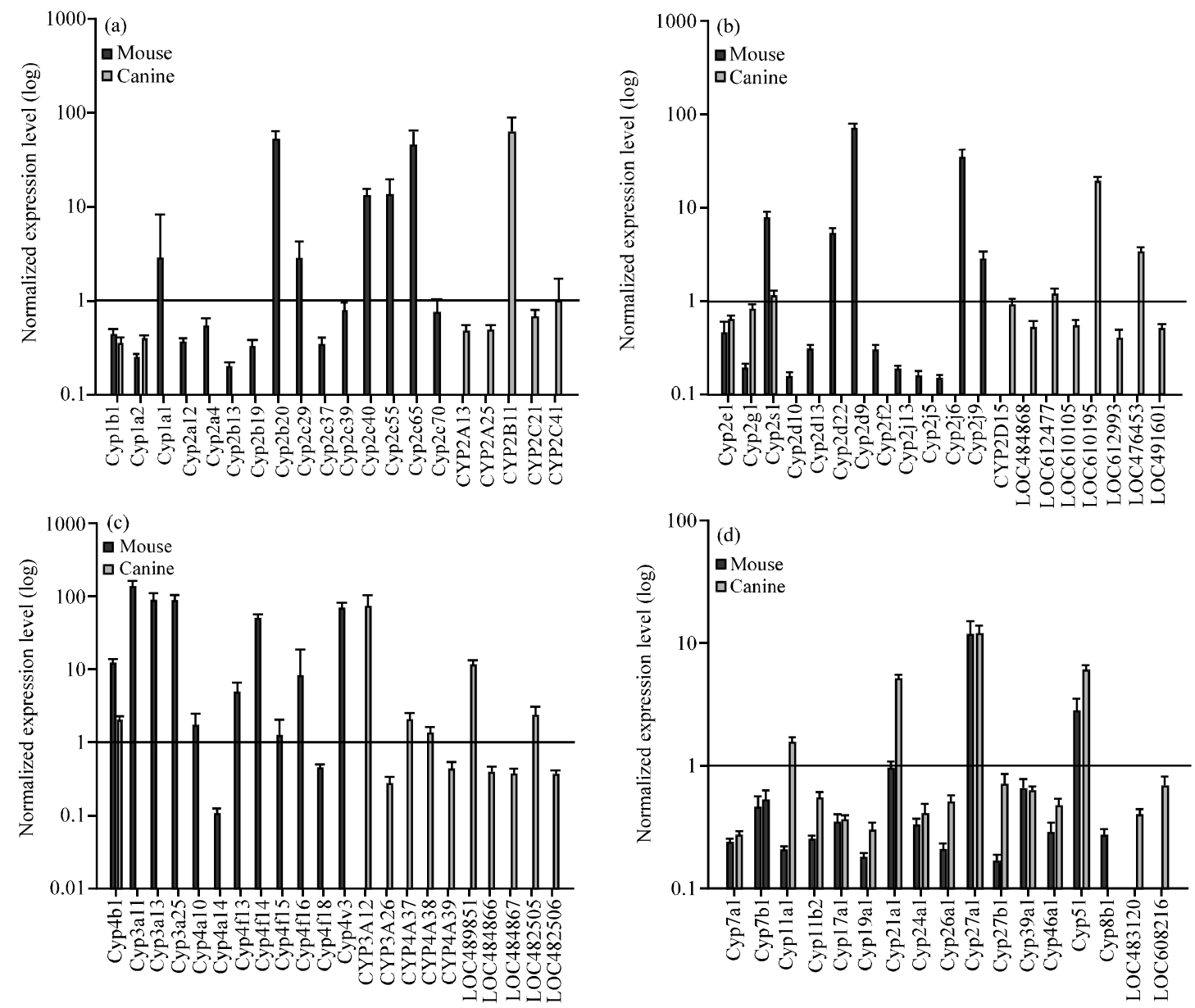

Fig. 1a-d: Comparative gene expression for various cytochrome p450s in the intestine of dogs and mice $(n=4)$

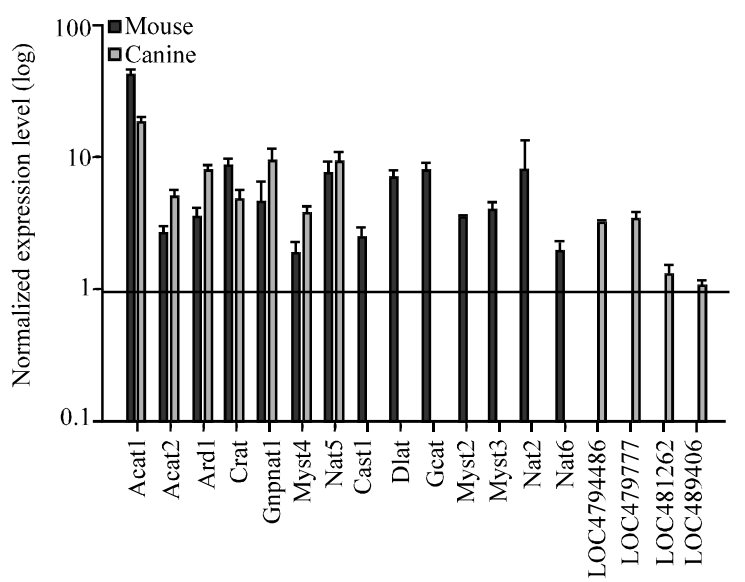

Fig. 2: Comparative gene expression for various acetyltransferases in the intestine of dogs and mice $(n=4)$

acetyltransferase, LOC479486: similar to polyamine Nacetyltransferase, LOC479777: similar to MYST histone acetyltransferase 1, LOC481262: similar to 2-amino-3- ketobutyrate coenzyme A ligase, mitochondrial precursor, LOC489406: similar to dihydrolipoamide S-acetyltransferase).

Acyltransferases: Figure 3 shows the acyltransferase expression levels. ACAA1 and 2, AGPAT1, DGAT1 and 2 and LRAT were commonly expressed and their orders of expressions were very similar in both species. Expression levels were measured by the intensity of hybridization signal using GeneChip array. (ACAA 1,2: acetylCoenzyme A acyltransferase 1, 2, AGPAT 1,3: 1acylglycerol-3-phosphate O-acyltransferase 1,3, BAAT: bile acid-Coenzyme $\mathrm{A}$ : amino acid $\mathrm{N}$-acyltransferase, DGAT 1, 2: diacylglycerol O-acyltransferase 1, 2, LRAT: lecithin-retinol acyltransferase, GNPAT: glyceronephosphate O-acyltransferase, SOAT1: sterol O-acyltransferase 1, Gpam: glycerol-3-phosphate acyltransferase, mitochondrial, LOC475572: similar to putative lysophosphatidic acid acyltransferase, LOC475658: similar to O-acyltransferase (membrane bound) domain containing 2, LOC477023: similar to 


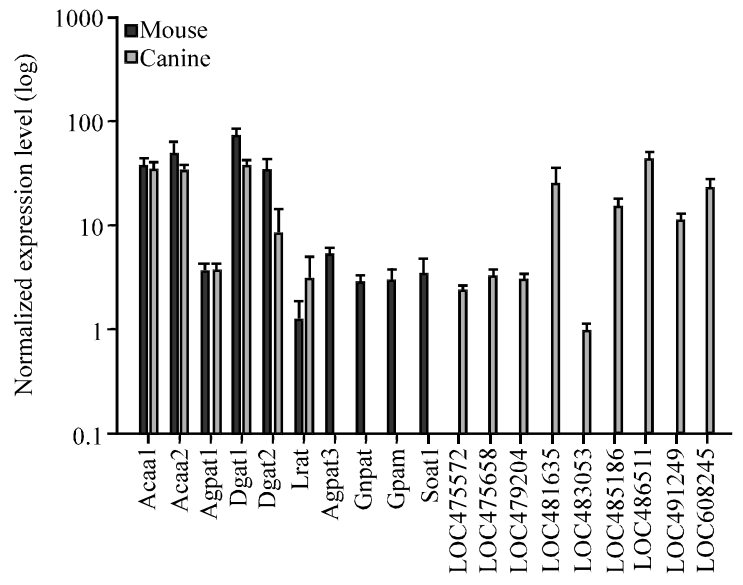

Fig. 3: Comparative gene expression for various acyltransferases in the intestine of dogs and mice $(\mathrm{n}=4)$

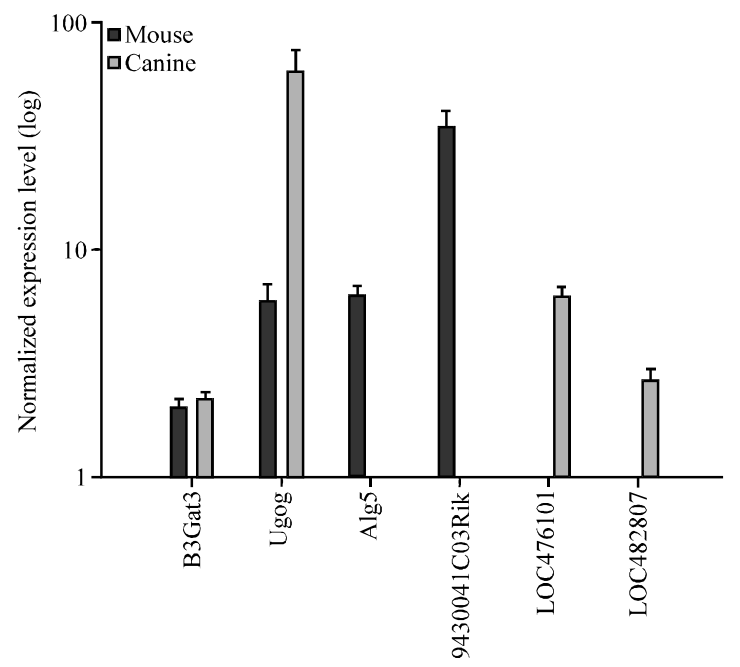

Fig. 4: Comparative gene expression for various glucuronosyltransferases in the intestine of dogs and mice $(\mathrm{n}=4)$

3-ketoacyl-CoA thiolase, peroxisomal precursor, LOC479204: similar to Dihydroxyacetone phosphate acyltransferase (DHAP-AT), LOC483053: similar to Glycerol-3-phosphate acyltransferase, mitochondrial precursor (GPAT), LOC485186: similar to monoacylglycerol O-acyltransferase 2, LOC486511: similar to sterol O-acyltransferase 2, LOC491249: similar to 1-acylsn-glycerol-3-phosphate acyltransferase beta (1-AGP acyltransferase 2), LOC608245: similar to monoacylglycerol O-acyltransferase 3).

Glucuronosyltransferases: UDP-Glucuronosyltransferase (UGT), one of the well-known phase II drug metabolizing enzymes, mediates conjugation of glucuronic acid to drugs. The present findings (Fig. 4) showed that both

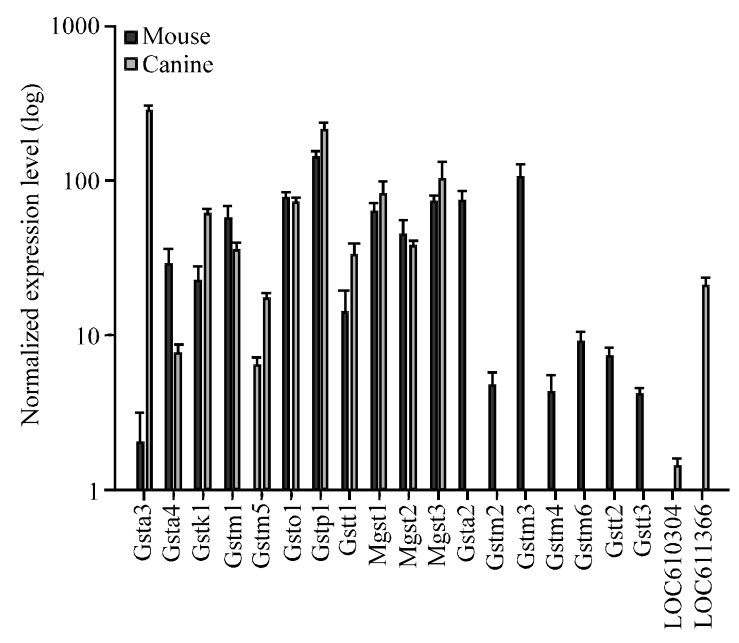

Fig. 5: Comparative gene expression for various glutathione S-transferases in the intestine of dogs and mice $(n=4)$

B3GAT3 and UGCG were commonly expressed. However, the expressions of UGCG in dogs were much higher than in mouse. Expression levels were measured by the intensity of hybridization signal using GeneChip array. (Alg5: asparagine-linked glycosylation 5 homolog (yeast, dolichyl-phosphate beta-glucosyl-transferase), Ugcg: UDP-glucose ceramide glucosyltransferase, 9430041C03Rik: UDP-glucuronosyltransferase 2 family, member 5, B3gat1: beta-1, 3-glucuronyltransferase 1 (glucuronosyltransferase P), B3Gat3: beta-1, 3glucuronyltransferase 3 (glucuronosyltransferase I) Ugt8: UDP-glucuronosyltransferase 8, LOC476101: similar to UDP-glucose ceramide glucosyltransferase-like 1 isoform 1, UGCG; LOC481666: UDP-glucose ceramide glucosyltransferase).

Glutathione S-transferases: These enzymes have been subdivided into two major classes, Glutathione STransferase (GSTs) and Microsomal Glutathione STransferase (MGSTs). Figure 5 shows the gene expression from of various glutathione S-transferases in the dogs and mice duodenums. GSTA3 and 4, GSTK1, GSTM1 and 5, GSTO1, GSTP1, GSTT1 and MGST1 3 were expressed in both species. Among them, GSTA3, GSTK1 and GSTM5 were highly expressed in dogs compared to mice (t-test, $\mathrm{p}<0.01$ ). Expression levels were measured by the intensity of hybridization signal using GeneChip array. (GSTA2, 3, 4: glutathione S-transferase, alpha 2, 3, GSTK1: glutathione S-transferase, kappa 1, GSTM1, 2, 3, 4, 5, 6: glutathione S-transferase, mu 1, 2, 3, 4, 5, 6, GSTO1: glutathione S-transferase, omega 1, GSTP1: glutathione Stransferase, pi 2, GSTT1, 2, 3: glutathione S-transferase, theta 1, 2, 3 MGST1, 2, 3: microsomal glutathione Stransferase 1, 2, 3, GSTA3: similar to glutathione S- 


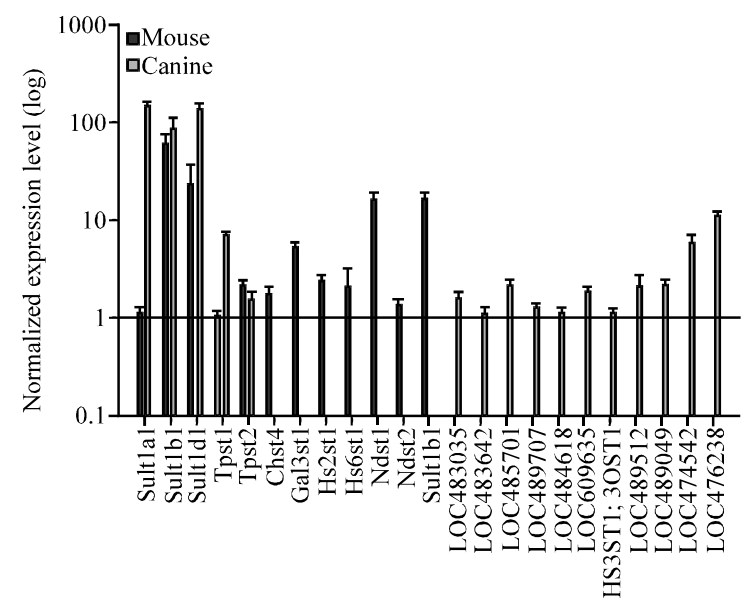

Fig. 6: Comparative gene expression for various sulfotransferases in the intestine of dogs and mice $(\mathrm{n}=4)$

transferase A3-3 (GST class-alpha), LOC474939: similar to glutathione S-transferase A4, LOC475518: similar to glutathione S-transferase kappa 1 (GST 13-13), LOC475518; LOC609921: similar to glutathione Stransferase kappa 1 (GST 13-13), LOC476006: similar to glutathione S-transferase, pi 1, LOC476078: similar to microsomal glutathione S-transferase 2 (Microsomal GST2), LOC477558: similar to glutathione S-transferase theta 2 (GST class-theta 2), LOC477683: similar to microsomal glutathione S-transferase 1 (Microsomal GST-1), LOC477813: similar to glutathione-S-transferase omega 1, LOC 478990; LOC609050: similar to microsomal glutathione S-transferase 3, LOC479911: similar to glutathione-Stransferase, mu 5, LOC479912: similar to glutathione Stransferase M1 isoform 2, LOC610304: similar to glutathione S-transferase 5.7 (GST 5.7), LOC611366: similar to glutathione S-transferase P (GST 7-7)).

Sulfotransferases: Sulfotransferase catalyzes the transfer of sulfate group to the hydroxyl group of drug compounds. In this study, SULT1A1, SULT1B1, SULT1D1, TPST1 and TPST2 were expressed in both species and a trend of higher expression in dogs were observed from SULT1A1, 1D1 and TPST1 (t-test, p<0.01) (Fig. 6). Expression levels were measured by the intensity of hybridization signal using GeneChip array. (CHST4: carbohydrate (chondroitin 6/keratan) sulfotransferase 4, GAL3ST1: galactosylceramide sulfotransferase, HS2ST1: heparan sulfate 2-O-sulfotransferase 1, HS3ST1: heparan sulfate (glucosamine) 3-O-sulfotransferase 1, HS6ST1: heparan sulfate 6-O-sulfotransferase 1. NDST1,2: Ndeacetylase/N-sulfotransferase (heparan glucosaminyl) 1 , 2, SULT: sulfotransferase, TPST1, 2: protein-tyrosine sulfotransferase 1, 2, HS3ST1; 3OST1: heparan sulfate (glucosamine) 3-O-sulfotransferase 1, LOC474542: similar to Sulfotransferase 1C2, LOC476238: similar to uronyl-2sulfotransferase, LOC483035: similar to CCAAT/enhancer binding protein zeta, LOC483642: similar to carbohydrate (keratan sulfate Gal-6) sulfotransferase 1, LOC484618: similar to carbohydrate (chondroitin 4) sulfotransferase 13, LOC485701: similar to carbohydrate (Nacetylglucosamine-6-O) sulfotransferase 2, LOC489049: similar to heparin sulfate, acetylase $\mathrm{N}$-sulfotransferase $(\mathrm{N}$ HSST), LOC489512; LOC489516: similar to heparan sulfate D-glucosaminyl 3-O-sulfotransferase 3A1, LOC489707: similar to carbohydrate ( $\mathrm{N}$-acetylglucosamine 6-O) sulfotransferase 6, LOC609635: similar to carbohydrate sulfotransferase 12).

\section{DISCUSSION}

Recent targeting specific tissue genes such as intestinal transporters or enzymes has shown a potential to greatly enhance the understanding for pharmacokinetics including bioavailability and metabolic clearance for oral drug molecules (Rushmore and Kong, 2002; Tsuji, 2002; Beaumont, 2003; Landowski et al., 2003; Mizuno et al., 2003; Takara et al., 2003). It is now accepted that the drug absorption process from intestine is highly associated with the functional gene expression of intestinal transporters and metabolizing enzymes (Landowski et al., 2003). The completion of the DNA sequencing of human, mouse, dog and rat genomes and knowledge of cross-species gene homologies enables the studies of differential gene expression in animal models (Fang et al., 2005).

Dog is a very important animal species not only as a laboratory model but also as a target animal in veterinary medicine. The information regarding DNA expression in dogs' intestine has not been sufficiently known. The DNA microarray technology is a high-throughput method for gaining information on gene function. Due to the high number of generated data points, computational tools are essential in microarray data analysis and mining to grasp knowledge from experimental results. Some of the methodology has been focused on gene expression intensity measures, microarray data normalization and statistical validation of differential expression (Wu et al., 2007).

In this study, computational approaches for GeneChip expression measures, data normalization and statistical validation were performed using GeneSpring 7.2 software. In microarray analysis, one should exclude non-informative probe sets from the original dataset before getting to the statistical validation of the differential expression. This step can be achieved by performing various filtering procedures. The stringency of the filtering procedure could strongly affect (in a positive or a negative manner) the final results as it can cause the loss of differentially expressed genes or increase the 
number of false positives contaminating the final results. In this study, filtering by expression was used to find gene with intraspecise similarity and interspecies difference. Furthermore using the standard deviations of probe set hybridization, all probe sets showing low hybridization quality were removed. The intensity values obtained using RMA/quantile normalization was subsequently coupled to the filtered genes and were used for statistical validation.

A variety of phase I and phase II enzymes are expressed in the small intestine, of which cytochrome $\mathrm{P} 450 \mathrm{~s}$ is the most prominent. Although, the cytochrome P450 enzymes are predominantly found in the liver, the intestinal cytochrome P450s also deserve special mechanism for modulating drug bioavailability because this organ is the main contributor of prehepatic clearance of drugs (Kaminsky and Fasco, 1991; Doherty and Charman, 2002). Considerable recent progress has been made in the identification and characterization of small intestinal CYP450s, particularly in rats (Zhang et al., 2007) and humans (Ding and Kaminsky, 2003). However, the expression of these enzymes in mice and dogs is less characterized than those in rats and human. Human have shown a dominant expression of $3 \mathrm{~A} 4,2 \mathrm{D} 6,2 \mathrm{C} 8,2 \mathrm{C} 9$ and $1 \mathrm{~A} 2$ and rats have shown $1 \mathrm{~A} 1,3 \mathrm{~A} 1,2 \mathrm{~B} 1,2 \mathrm{C} 6 / 11$ and $2 \mathrm{D} 1$ in intestine (Lindell et al., 2003). Unlike these species, the study reveals dominant expression of $3 \mathrm{~A} 12,2 \mathrm{~B} 11,2 \mathrm{~J} 2$ and $3 \mathrm{~A} 4$ in dogs and $3 \mathrm{~A} 11,3 \mathrm{~A} 12$ and $3 \mathrm{~A} 15$ in mice. Previous reports (Prueksaritanont et al., 1996; Emoto et al., 2000) also exhibited a dominant presence of CYP3A family enzymes in dog and mouse intestine. In the human intestine, CYP3A4 is a predominant cytochrome P450 enzyme and plays a significant role in the first-pass metabolism of 50-70\% of marketed drugs (Wacher et al., 1998). However, these species including rats, mice and dogs do not show a primary expression of CYP3A4 in intestine while other cytochrome $\mathrm{P} 450$ family or isoforms are predominantly expressed. This may indicate that prehepatic metabolism of oral drugs is extensively different by species and substrate structures.

Acetylation is metabolic pathway of aromatic amine. The hydorylamine metabolites of aromatic amines can also be a substrate for acetyl transferases because acetate is a better leaving group than hydroxide. The most common aryl amine drugs are the. In the study, ACAT1, ACAT2, ARD1, CRAT, GNPNAT1, MYST4 andNAT5 were greatly expressed in both dogs and mice. Among the acetyltransferases, ACAT1 was dominant in both species. No tendency of expression differences between the two species was observed. The glucosyltransferases catalyze the addition of a glucurin acid moiety to a nucleophilic substrate using UDP-glucuronic acid as cofactor. As with the CYPs, UGT superfamily possesses a large multiplicity of forms and wide tissue distribution (Guillemette, 2003). Gastrointestinal UGTs (e.g., UGT1A1, UGT1A7-10) may contribute to the low oral bioavailability of ethinyl estradiol (Ebner et al., 1993) and raloxifene (Hochner-Celnikier, 1999; Kemp et al., 2002) and to the first-pass glucuronidation of SN-38 (Khanna et al., 2000) and troglitazone (Watanabe et al., 2002). In this study, B3GAT3 and UGCG were considerably expressed in dogs and mice intestine however, the expression of UGCG in dogs was much higher than that in mouse.

Glutathione S-transferase families are commonly implicated in the detoxification or bioactivation of environmental toxins and carcinogens and some chemotherapeutic agents. Intestinal GSTA has been shown to conjugate busload with an intrinsic clearance comparable to liver (Gibbs et al., 1998). This study showed the gene expression form of various glutathione S-transferases in dogs and mice duodenum. GSTA3 and 4, GSTK1, GSTM 1 and 5, GSTO1, GSTP1, GSTT1, MGST1, 2 and 3 were expressed in both species and among them, GSTA3, GSTK1 and GSTM5 were significantly and highly expressed more in dogs compared to mice.

Although, sulfation along with other phase II metabolism is generally perceived as a predominant detoxification pathway, there are a number of importances where sulfate conjugates are more biologically active than the parent molecules. The extensive first-pass loss of ethinyl estradiol is also attributed to Sulfotransferases (SULTs) in the intestine (SULT1E1, SULT2A1) (Glatt et al., 2001). Likewise, intestinal SULT1A3 has been implicated in the low oral bioavailability of the $\beta$-adrenergic agents, isoproterenol and terbutaline (Glatt et al., 2001). This study shows that SULT1A1, SULT 1B1, SULT 1D1, TPST1 and TPST2 were expressed in both species but trends of higher expressions of SULT1A1, SULT 1D1 and TPST1 in dogs were observed.

\section{CONCLUSION}

In this study, GeneChip ${ }^{\circledR}$ analysis indicated the marked differences in expression profiles of metabolizing enzymes between dogs and mice duodenum. As a model animal, gene information of mouse has been explored in detail but that of dogs is insufficient to be compared to gene directly. However, significant differences in gene expression levels in dogs and mouse duodenums were observed. The gene expression profiling of this two important animal species is a valuable new tool for investigating the oral drug prehepatic metabolism in vitro and in vivo. Also the dataset provides a baseline for comparison of normal duodenum in the two important species as model and/or target animals. 


\section{ACKNOWLEDGEMENT}

This study was supported by the BK21 Program of Ministry of Education, Republic of Korea.

\section{REFERENCES}

Amidon, G.L., P.J. Sinko and D. Fleisher, 1988. Estimating human oral fraction dose absorbed: A correlation using rat intestinal membrane permeability for passive and carrier-mediated compounds. Pharm. Res., 5: 651-654.

Anzenbacher, P. and E. Anzenbacherova, 2001. Cytochromes P450 and metabolism of xenobiotics. Cell. Mol. Life Sci., 58: 737-747.

Beaumont, K., 2003. The Importance of Gut Wall Metabolism in Determining Drug Bioavailability. In: Drug Bioavailability: Estimation of Solubility, Permeability and Absorption, Waterbeemd, H.V.D., H Lennernas and P. Artursson (Eds.). Wiley-VCH Verlag, New York, pp: 311-328.

Brown, P.O. and D. Botstein, 1999. Exploring the new world of the genome with DNA microarrays. Nat. Genet., 21: 33-37.

Che, M., D.F. Ortiz and I.M. Arias, 1995. Primary structure and functional expression of a cDNA encoding the bile canalicular, purine-specific $\mathrm{Na}^{+}$-nucleoside cotransporter. J. Biol. Chem., 270: 13596-13599.

Ding, X. and L.S. Kaminsky, 2003. Human extrahepatic cytochromes P450: Function in xenobiotic metabolism and tissue-selective chemical toxicity in the respiratory and gastrointestinal tracts. Annu. Rev. Pharmacol. Toxicol., 43: 149-173.

Doherty, M.M. and W.N. Charman, 2002. The mucosa of the small intestine: how clinically relevant as an organ of drug metabolism?. Clin. Pharmacokinet., 41: $235-253$.

Ebner, T., R.P. Remmel and B. Burchell, 1993. Human bilirubin UDP-glucuronosyltransferase catalyzes the glucuronidation of ethinylestradiol. Mol. Pharmacol., 43: 649-654.

Emoto, C., H. Yamazaki, S. Yamasaki, N. Shimada, M. Nakajima and T. Yokoi, 2000. Characterization of cytochrome P450 enzymes involved in drug oxidations in mouse intestinal microsomes. Xenobiotica, 30: 943-953.

Fang, H., W. Tong, R. Perkins, L. Shi and H. Hong et al., 2005. Bioinformatics approaches for cross-species liver cancer analysis based on microarray gene expression profiling. BMC Bioinf., 15: S6-S6.
Gibbs, J.P., J.S. Yang and J.T. Slattery, 1998. Comparison of human liver and small intestinal glutathione S-transferase-catalyzed busulfan conjugation in vitro. Drug Metab. Dispos., 26: 52-55.

Glatt, H., H. Boeing, C.E. Engelke, L. Ma and A. Kuhlow et al., 2001. Human cytosolic sulphotransferases: genetics, characteristics, toxicological aspects. Mutat. Res., 482: 27-40.

Guillemette, C., 2003. Pharmacogenomics of human UDP-glucuronosyltransferase enzymes. Pharm. J., 3: $136-158$.

Hochner-Celnikier, D., 1999. Pharmacokinetics of raloxifene and its clinical application. Eur. J. Obstet. Gynecol. Reprod. Biol., 85: 23-29.

Kaminsky, L.S. and M.J. Fasco, 1991. Small intestinal cytochromes P450. Crit. Rev. Toxicol., 21: 407-422.

Kemp, D.C., P.W. Fan and J.C. Stevens, 2002. Characterization of raloxifene glucuronidation in vitro: Contribution of intestinal metabolism to presystemic clearance. Drug Metab. Dispos., 30: 694-700.

Khanna, R., C.L. Morton, M.K. Danks and P.M. Potter, 2000. Proficient metabolism of irinotecan by a human intestinal carboxylesterase. Cancer Res., 60: 4725-4728.

Landowski, C.P., D. Sun, D.R. Foster, S.S. Menon, J.L. Barnett et al., 2003. Gene expression in the human intestine and correlation with oral valacyclovir pharmacokinetic parameters. J. Pharmacol. Exp. Ther., 306: 778-786.

Lindell, M., M. Lang and H. Lennernas, 2003. Expression of genes encoding for drug metabolising cytochrome P450 enzymes and P-glycoprotein in the rat small intestine; comparison to the liver. Eur. J. Drug Metab. Pharmacokinet, 28: 41-48.

Mizuno, N., T. Niwa, Y. Yotsumoto and Y. Sugiyama, 2003. Impact of drug transporter studies on drug discovery and development. Pharmacol. Rev., 55: 425-461.

Oh, D.M., P.J. Sinko and G.L. Amidon, 1993. Characterization of the oral absorption of some $\beta$-lactams: Effect of the $\alpha$-amino side chain group. J. Pharm. Sci., 82: 897-900.

Prueksaritanont, T., L.M. Gorham, J.H. Hochman, L.O. Tran and K.P. Vyas, 1996. Comparative studies of drug-metabolizing enzymes in dog, monkey and human small intestines and in CaCO-2 cells. Drug Metab. Dispos., 24: 634-642.

Rushmore, T.H. and A.N. Kong, 2002. Pharmacogenomics, regulation and signaling pathways of phase I and II drug metabolizing enzymes. Curr. Drug Metab., 3: $481-490$. 
Shin, H.C., H.R. Kim, H.J. Cho, H. Yi, S.M. Cho et al., 2009. Comparative gene expression of intestinal metabolizing enzymes. Biopharm. Drug Dispos., 30: 411-421.

Starkey, M.P., T.J. Scase, C.S. Mellersh and S. Murphy, 2005. Dogs really are man's best friend-canine genomics has applications in veterinary and human medicine. Brief Funct. Genomic Proteomic, 4: 112-128.

Takara, K., N. Ohnishi, S. Horibe and T. Yokoyama, 2003. Expression profiles of drug-metabolizing enzyme $\mathrm{CYP}_{3} \mathrm{~A}$ and drug efflux transporter multidrug resistance 1 subfamily mRNAS in small intestine. Drug Metab. Dispos., 31: 1235-1239.

Tsuji, A., 2002. Transporter-mediated drug interactions. Drug Metab. Pharmacokinet, 17: 253-274.

Wacher, V.J., J.A. Silverman, Y. Zhang and L.Z. Benet, 1998. Role of P-glycoprotein and cytochrome P450 $3 \mathrm{~A}$ in limiting oral absorption of peptides and peptidomimetics. J. Pharm. Sci., 87: 1322-1330.
Wang, W., J. Jiang, C.E. Ballard and B. Wang, 1999. Prodrug approaches to the improved delivery of peptide drugs. Curr. Pharm. Des., 5: 265-287.

Watanabe, Y., M. Nakajima and T. Yokoi, 2002. Troglitazone glucuronidation in human liver and intestine microsomes: High catalytic activity of UGT1A8 and UGT1A10. Drug Metab. Dispos., 30: 1462-1469.

$\mathrm{Wu}$, B., J. Wang and M. Wang, 2007. Software development in data analysis and mining for cDNA microarray. Sheng Wu Yi Xue Gong Cheng Xue Za Zhi, 24: 1394-1397.

Zhang, W., T.M.C. Tan and L.Y. Lim, 2007. Impact of curcumin-induced changes in P-glycoprotein and $\mathrm{CYP}_{3} \mathrm{~A}$ expression on the pharmacokinetics of peroral celiprolol and midazolam in rats. Drug Metab. Dispos., 35: 110-115. 